\title{
Functional drug-target-disease network analysis of gene-phenotype connectivity for curcumin in hepatocellular carcinoma
}

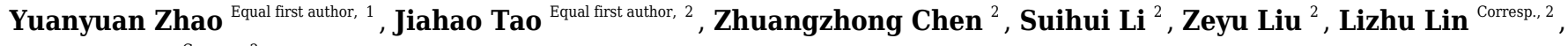 \\ Linzhu Zhai ${ }^{\text {Corresp. } 2}$ \\ ${ }^{1}$ State Key Laboratory of Oncology in South China, Sun Yat-sen University Cancer Center, Guangzhou, Guangdong, P. R. China \\ Cancer Center, the First Affiliated Hospital of Guangzhou University of Chinese Medicine, Guangzhou, Guangdong, P. R. China \\ Corresponding Authors: Lizhu Lin, Linzhu Zhai \\ Email address: linlizhu@gzucm.edu.cn, linzhuzhai@163.com
}

Background: The anti-tumor properties of curcumin have been demonstrated for many types of cancer. However, a systematic functional and biological analysis of its target proteins has yet to be fully documented. The aim of this study was to explore the underlying mechanisms of curcumin and broaden the perspective of targeted therapies. Methods: Direct protein targets (DPTs) of curcumin were searched in the DrugBank database. Using the STRING database, the interactions between curcumin and DPTs and indirect protein targets (IPTs) weres documented. The protein-protein interaction (PPI) network of curcumin-mediated proteins was visualized using Cytoscape. Kyoto Encyclopedia of Genes and Genomes (KEGG) pathway enrichment analysis was performed for all curcumin-mediated proteins. Furthermore, the cancer targets were searched in the Comparative Toxicogenomics Database (CTD). The overlapping targets were studied using Kaplan-Meier analysis to evaluate cancer survival. Further genomic analysis of overlapping genes was conducted using the cBioPortal database. Lastly, MTT, quantitative polymerase chain reaction (qPCR), and western blot (WB) analysis were used to validate the predicted results on hepatocellular carcinoma (HCC) cells. Results: A total of five DPTs and 199 IPTs were found. These protein targets were found in 121 molecular pathways analyzed via KEGG enrichment. Based on the antitumor properties of curcumin, two pathways were selected, including pathways in cancer (36 genes) and HCC (22 genes). Overlapping with 505 HCC-related gene sets identified in CTD, five genes (TP53, RB1, TGFB1, GSTP1, and GSTM1) were finally identified. High mRNA levels of TP53, RB1, and GSTM1 indicated a prolonged overall survival (OS) in HCC, whereas elevated mRNA levels of TGFB1 were correlated with poor prognosis. The viability of both HepG2 cells and Hep3B cells was significantly reduced by curcumin at concentrations of 20 or $30 \mu \mathrm{M}$ after 48 or $72 \mathrm{~h}$ of culture. At a concentration of $20 \mu \mathrm{M}$ curcumin cultured for 48h, the expression of TGFB1 and GSTP1 in Hep3B cells was reduced significantly in qPCR analysis, and reduced TGFB1 protein expression was also found in Hep3B cells. 
1 Functional drug-target-disease network analysis of gene2 phenotype connectivity for curcumin in hepatocellular 3 carcinoma

4

5 Yuanyuan Zhao ${ }^{1 \dagger}$, Jiahao $\mathrm{Tao}^{2 \dagger}$, Zhuangzhong $\mathrm{Chen}^{2}$, Suihui $\mathrm{Li}^{2}$, Zeyu Liu ${ }^{2}$, Lizhu Lin ${ }^{2}$ and 6 Linzhu Zhai ${ }^{2}$

7

$8{ }^{1}$ Sun Yat-sen University Cancer Center; State Key Laboratory of Oncology in South China; 9 Collaborative Innovation Center for Cancer Medicine, Guangzhou, Guangdong, P. R. China;

$10{ }^{2}$ Cancer Center, the First Affiliated Hospital of Guangzhou University of Chinese Medicine, 11 Guangzhou, Guangdong, P. R. China;

13 Corresponding Author:

14 Lizhu Lin ${ }^{2}$;Linzhu Zhai ${ }^{2}$

15 Jichang Road 16\#, Guangzhou, Guangdong, 510080, P. R. China

16 Email address: linzhuzhai@163.com

17 Tel: +86-020-36591419

$\dagger$ These authors have contributed equally to this work and share first authorship 
39

40

41

42

43

44

45

46

47

48

49

50

51

52

53

54

55

56

57

58

59

60

61

62

63

64

65

66

67

68

69

70

71

72

73

74

75

76

77

78

\section{Abstract}

Background: The anti-tumor properties of curcumin have been demonstrated for many types of cancer. However, a systematic functional and biological analysis of its target proteins has yet to be fully documented. The aim of this study was to explore the underlying mechanisms of curcumin and broaden the perspective of targeted therapies.

Methods: Direct protein targets (DPTs) of curcumin were searched in the DrugBank database. Using the STRING database, the interactions between curcumin and DPTs and indirect protein targets (IPTs) weres documented. The protein-protein interaction (PPI) network of curcuminmediated proteins was visualized using Cytoscape. Kyoto Encyclopedia of Genes and Genomes (KEGG) pathway enrichment analysis was performed for all curcumin-mediated proteins. Furthermore, the cancer targets were searched in the Comparative Toxicogenomics Database (CTD). The overlapping targets were studied using Kaplan-Meier analysis to evaluate cancer survival. Further genomic analysis of overlapping genes was conducted using the cBioPortal database. Lastly, MTT, quantitative polymerase chain reaction (qPCR), and western blot (WB) analysis were used to validate the predicted results on hepatocellular carcinoma (HCC) cells.

Results: A total of five DPTs and 199 IPTs were found. These protein targets were found in 121 molecular pathways analyzed via KEGG enrichment. Based on the anti-tumor properties of curcumin, two pathways were selected, including pathways in cancer (36 genes) and HCC (22 genes). Overlapping with 505 HCC-related gene sets identified in CTD, five genes (TP53, RB1, TGFB1, GSTP1, and GSTM1) were finally identified. High mRNA levels of TP53, RB1, and GSTM1 indicated a prolonged overall survival (OS) in HCC, whereas elevated mRNA levels of TGFB1 were correlated with poor prognosis. The viability of both HepG2 cells and Hep3B cells was significantly reduced by curcumin at concentrations of 20 or $30 \mu \mathrm{M}$ after 48 or $72 \mathrm{~h}$ of culture. At a concentration of $20 \mu \mathrm{M}$ curcumin cultured for $48 \mathrm{~h}$, the expression of TGFB1 and GSTP1 in Hep3B cells was reduced significantly in qPCR analysis, and reduced TGFB1 protein expression was also found in Hep3B cells.

67

(1)

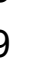

1

2




\section{Introduction}

80

81

82

83

84

85

86

87

88

89

90

91

92

93

94

95

96

97

98

99

100

101

102

103

104

105

106

107

108

109

110

111

112

113

114

115

116

117

118

Primary liver cancer is the sixth most common cancer and the second largest cause of cancer mortality in the world (Ferlay et al. 2015). Hepatocellular carcinoma (HCC) constitutes approximately $80 \%$ of all primary liver cancer (McGlynn et al. 2015). The main causes of HCC are hepatitis $\mathrm{B}$ and $\mathrm{C}$ virus infection, alcoholic liver disease, non-alcoholic fatty liver diseases related to obesity and/or diabetes, aflatoxin B1 contamination of food, and less frequently, genetic predisposition. The incidence of HCC is increasing worldwide, mostly due to the spread of chronic infections with hepatitis $\mathrm{B}$ and $\mathrm{C}$ viruses. Most of these risk factors cause persistent inflammation in the liver, leading to the formation of liver cirrhosis and then HCC.

Curcumin, also known as diferuloylmethane, is the active ingredient of the dietary spice occurring in the rhizomes of Curcuma longa, a plant belonging to the ginger family (Figure 2A). Extensive studies investigating curcumin over the past few decades revealed the health benefits of curcumin, including anticancer, anti-inflammatory, antioxidant, and hypoglycemic effects (Prasad et al. 2014). In recent years, curcumin has been increasingly recognized for its anti-tumor and chemopreventive properties, especially in gastrointestinal tumors (Huminiecki et al. 2017). For example, curcumin has shown chemopreventive effects in animal models of colon cancer (Kawamori et al. 1999), stomach cancer (Huang et al. 1994), and HCC (Chuang et al. 2000). Curcumin has been reported to decrease cell growth and induce apoptosis mainly through the inhibition of nuclear factor kappa-B (NFKB) (Ghasemi et al. 2019). Recent reports also indicate the curcumin could decrease tumor cell viability by inhibiting the activity of ATP synthase in isolated mitochondrial membranes which leads to a dramatic drop of ATP and a reduction of oxygen consumption (Bianchi et al. 2018). In addition, DYRK2 has been identified as a direct target of curcumin. By diminishes DYRK2-mediated 26S proteasome phosphorylation in cells, curcumin leading to reduced proteasome activity and inhibited cell proliferation (Banerjee et al. 2018). Several phase I and II clinical trials with curcumin have been conducted for the treatment of different types of cancer (Gupta et al. 2013; Hatcher et al. 2008).

Recent studies using data mining techniques for the analysis of specific bioinformatics domains have made great progress (Lan et al. 2018). Accordingly, a strategy to systematically explore the rich data publicly available and the underlying connectivity between gene and phenotype mediated by curcumin should be helpful. With advances in genomics, data network analysis has been used to effectively analyze candidate genes linked to experimentally verifiable pathways via mining of web-accessible, open portal databases (Hsieh et al. 2016). In addition, an in-depth analysis of the databases may uncover, previously unknown relationships between drugs, targets, and cancers. These results can be used to generate authentic and rational leads for further investigations. Recently, the combination of related databases including DrugBank, STRING, cBio Cancer Genomics Portal (cBioPortal), and Comparative Toxicogenomics Database (CTD) has been utilized for drug-target-cancer network analysis(Fu et al. 2019; Zhang et al. 2018). In this study, DrugBank was employed to broadly analyze curcumin and drug-target data to obtain related direct protein targets (DPTs). Interactions between DPTs and indirect protein targets (IPTs) were

Peer) reviewing PDF | (2021:05:61606:2:0:NEW 21 Sep 2021) 
119 predicted with the STRING database. The readout DPTs and IPTs were further analyzed for 120 functionality via Kyoto Encyclopedia of Genes and Genomes (KEGG) pathway enrichment 121 analysis with STRING. The pivotal genes were obtained via intersections of the CTD and STRING 122 databases, and the genomic alterations were investigated using the cBioPortal database. Our 123 findings provide information to obtain a better understanding of the anti-tumor mechanisms of 124 curcumin and identify new targets in the treatment of HCC by curcumin.

125

126

\section{7}

128

129

130

131

132

133

134

135

136

137

138

139

140

141

142

143

144

145

146

147

148

149

150

151

152

153

154

155

156

157

158

\section{Materials and Methods}

Search for DPTs of curcumin

The DrugBank database (https://www.drugbank.ca) is a richly annotated resource that combines detailed drug data with comprehensive drug target and drug action information (Wishart et al. 2008). In this study, the latest release of DrugBank database (version 5.1.4) (Wishart et al. 2018) was employed to search for the interactions between curcumin and its DPTs and generate a curcumin-target network. The word "Curcumin" was searched as a keyword under drug classification entry.

PPI network generation

The STRING database (http://www.string-db.org/) provides a critical assessment and integration of protein-protein interactions, including direct (physical) as well as indirect (functional) associations (Szklarczyk et al. 2015). Using the latest STRING database (version 11.0)(Szklarczyk et al. 2019) search function, data underlying the interaction between DPTs and IPTs were first generated for curcumin by setting a minimum required interaction score of 0.5 and a maximum number of interactors of 50. The data were integrated into a curcumin-mediated network and visualized using Cytoscape (version 3.7.1) (Su et al. 2014), which is an open-source software platform for the visualization of complex networks and their integration with any attribute (Shannon et al. 2003).

KEGG pathway enrichment analysis and overlapping HCC genes Biochemical pathways linked to curcuminDPT/IPT interactions were navigated via the KEGG pathway enrichment analysis tool in the STRING database. The top 15 pathways with a False Discovery Rate (FDR) of less than 1.00E-10 were selected. To identify disease targets for HCC,"Hepatocellular carcinoma" was searched for in CTD (http://www.ctdbase.org/). CTD is a robust, publicly available database that aims to advance the understanding of environmental exposures and their impacts on human health. It provides manually curated information about chemical-gene/protein interactions, and chemical-disease and gene-disease relationships (Davis et al. 2019). Overlapping genes of HCC and cancer-related pathways and HCC targets were selected for further analysis. 
159 Exploring cancer genomics data linked to curcumin

160 The cBioPortal (https://www.cbioportal.org/) for Cancer Genomics provides a web resource for 161 exploration, visualization, and analysis of multidimensional cancer genomics data (Cerami et al. 162 2012; Gao et al. 2013). In this study, the screened genes from the foregoing investigation were 163 assessed in all HCC studies available in cBioPortal databases. Using the portal search function, 164 curcumin-related genes in HCC were evaluated for genomic alteration, network analysis was 165 performed, and mutual exclusivity or concurrent relationships between gene pairs of the same gene 166 set were identified. The study with the largest sample size was chosen to analyze the screened 167 proteins in cBioPortal databases to further interpret the results.

168

169

170

Analysis of curcumin-associated genomics datasets and HCC survival

171 The Kaplan-Meier method (http://kmplot.com/)(Nagy et al. 2018) was used to evaluate the overall survival (OS) in $364 \mathrm{HCC}$ samples with altered genes. OS was defined as the time to death. A twotailed $\mathrm{P}$ value of less than 0.05 was considered statistically significant.

173

174

Cell culture

175 HepG2 and Hep3B cells were purchased from Cell Cook (http://www.cellcook.com/). They were authenticated using short tandem repeat matching analysis and cultured in DMEM supplemented with $10 \% \mathrm{v} / \mathrm{v} \mathrm{FBS}$ and $1 \% \mathrm{v} / \mathrm{v}$ penicillin/streptomycin in $\mathrm{CO}_{2}$ incubator at $37^{\circ} \mathrm{C}$ and $95 \%$ relative humidity.

179

Cell viability test

181 The MTT (3-(4,5)-dimethylthiahiazo (-z-y1)-3,5-di-phenytetrazoliumromide) assay was used to assess cell viability. First, $100 \mu \mathrm{L}$ of cells (about 5000-10,000 cells) was transfused into a 96-well culture plate, which was then placed in a hatching house overnight for pre-culture $\left(37^{\circ} \mathrm{C}, 5 \% \mathrm{CO}_{2}\right)$. According to the drug concentration, all cells were divided into five groups. After pre-culture, 10 $\mu \mathrm{L}$ of drug solution was added to every well (the drug concentrations were $0,5,10,20,30 \mu \mathrm{M}$ ) and incubated at $37^{\circ} \mathrm{C}$. After that, $10 \mu \mathrm{L}$ MTT solution was added to each well at 24,48 , and $72 \mathrm{~h}$ and incubated at $37^{\circ} \mathrm{C}$ for $4 \mathrm{~h}$. The absorbance value of each hole at $450 \mathrm{~nm}$ was determined, and the cell vitality value was calculated. The cell vitality formula is as follows: Cell viability $\%=(O D$ in experimental group - OD in blank group)/(OD in control group - OD in blank group) $\times 100 \%$.

190

\section{Extraction of total RNA and reverse transcription-quantitative PCR (RT-qPCR)}

192 Cells of diffetent group were collected after being treated and cultured, and total RNA was extracted using TRIzol ${ }^{\circledR}$ reagent. Oligo (dT) primers and M-MLV reverse transcriptase were used to synthesize cDNA under the instruction of protocol. PCR amplification was performed on an RT-qPCR instrument with a GoTaq ${ }^{\circledR}$ qPCR Master mix. The relative gene expression of each sample was determined with the $2-\Delta \Delta \mathrm{Cq}$ method.

197

198

Western blot analysis 
199 Protein extractions were prepared with a modified RIPA buffer (Beyotime Institute of 200 Biotechnology) with $0.5 \%$ SDS. Proteinase inhibitor cocktail (Beyotime Institute of 201 Biotechnology) were involved in the whole process. Protein samples was separated using 202 SDS-PAGE, and then transferred to a PVDF membrane. The membranes were blocked with 5\% 203 BSA in TBS (containing $0.05 \%$ Tween-20) and incubated overnight with the primary antibodies. 204 After the membranes were washed, the membranes were incubated with horseradish peroxidase205 linked immunoglobulin G secondary antibody (Ms, Jackson ImmunoResearch) for $2 \mathrm{~h}$. The 206 membranes were developed using an enhanced chemiluminescence system (Thermo Scientific 207 Pierce).

208

209

\section{Results}

210

211 Characterization of curcumin DPTs

212 A flow chart of study steps was present in Figure 1. Drugbank was queried using curcumin as the 213 keyword to identify the bioactivities and to determine the DPTs of curcumin and retrieve relevant 214 information. The result showed an accession number of DB11672 and classified curcumin as a 215 highly pleiotropic molecule with anti-tumor, antibacterial, anti-inflammatory, hypoglycemic, 216 antioxidant, wound-healing, and antimicrobial activities (Gupta et al. 2013). Clinical data showed 217 that curcumin is undergoing clinical trials for colon cancer (NCT02724202), prostate cancer 218 (NCT03769766), breast cancer (NCT03980509), and lung cancer (NCT02321293). Subsequent 219 screening demonstrated five DPTs in human beings. Table 1 summarizes the five DPTs of 220 curcumin: PPARG, VDR, ABCC5, CBR1, and GSTP1. In addition, the interactions between five 221 DPTs were analyzed by STRING and illustrated in Figure 2B.

222

223

Characterization of curcumin IPTs and visualization of PPI network construction

Expanding the search using STRING database, a total of 204 target proteins of curcumin were detected, including 199 IPTs, which were related to five DPTs (Table S1). The dataset obtained was integrated to construct a biological network using Cytoscape. As shown in Figure 2C, 1962 PPI pairs were found in the network of curcumin-mediated proteins (Table S2). In this network, nodes represent proteins, and edges denote protein-protein associations. In addition, the node degree, which indicates the centrality of proteins, was calculated using CentiScaPe 2.2 (Table S3). As a result, 14 proteins including four DPT targets (GSTP1, VDR, CBR1, and PPARG) with a degree value $\geq 50$ are shown in Table 2 and displayed in Figure 2C with larger node sizes.

KEGG enrichment pathway analysis of proteins connected to curcumin To identify the functional features of curcumin-mediated targets, KEGG pathway enrichment analysis was performed using STRING. Target genes were found in 121 molecular pathways in KEGG enrichment (Table S4). As shown in Table 3 and Figure 2D, the top 15 KEGG pathways connected to curcumin-mediated proteins included metabolism of xenobiotics by cytochrome P450 (52 genes), chemical carcinogenesis (50 genes), drug metabolism-cytochrome P450 (44 
239

240

241

242

243

244

245

246

247

248

249

250

251

252

253

254

255

256

257

258

259

260

261

262

263

264

265

266

267

268

269

270

271

272

273

274

275

276

277

278

genes), retinol metabolism (32 genes), steroid hormone biosynthesis (30 genes), drug metabolismother enzymes (31 genes), pentose and glucuronate interconversion (22 genes), ascorbate and aldarate metabolism (20 genes), metabolic pathways (65 genes), glutathione metabolism (20 genes), porphyrin and chlorophyll metabolism (19 genes), arachidonic acid metabolism (18 genes), pathways in cancer (36 genes), HCC (22 genes), and fluid shear stress and atherosclerosis (19 genes). These KEGG enrichment pathways showed functional features of curcumin gene sets and indicated that curcumin-mediated proteins were mainly associated with basal metabolism and cancer-related pathways. Based on the anti-tumor properties of curcumin reported in diverse malignant tumors, two pathways were selected, including pathways in cancer (36 genes) and HCC (22 genes). This result suggested that HCC might be used as a phenotype connected to curcuminmediated proteins. In addition, to further identify disease targets for HCC, the phrase "Carcinoma, Hepatocellular" was searched in CTD, and a total of 505 genes with either a curated association or an inferred association via a curated chemical interaction with the disease were identified (Table S5). These genes were marked with "T," which indicates a gene that is or may be a therapeutic disease target, or "M," which denotes a gene that may be a disease biomarker or involved in the etiology of a disease. Five overlapping genes (TP53, RB1, TGFB1, GSTP1, and GSTM1) resulting from the intersections between pathways in cancer (36 genes), HCC pathway (22 genes), and targets for HCC by CTD (505 genes) were visualized using Venn diagrams and STRING (Figure $2 \mathrm{E}$ and $2 \mathrm{~F})$.

\section{Genetic alterations connected with curcumin-associated genes in HCC}

To further validate the link between curcumin-associated genes and HCC, cBioPortal databases were used to explore the five genes (TP53, RB1, TGFB1, GSTP1, and GSTM1) associated with curcumin in HCC. Seven HCC studies were included in cBioPortal (Ahn et al. 2014; Fujimoto et al. 2012; Harding et al. 2019; Pilati et al. 2014; Schulze et al. 2015; Zheng et al. 2018), and the five selected overlapping genes were queried. The results showed that $363(33 \%)$ of the 1135 samples in seven studies had alterations in one or more of these genes. Alterations ranged from $0.2 \%$ to $29 \%$ for gene sets submitted for analysis (Figure $3 \mathrm{~A}$ ). The five genes (TP53, RB1, TGFB1, GSTP1 and GSTM1) carried five gene pairs showing mutual exclusivity alterations, and another five gene pairs showed concurrent alterations (with TP53 and RB1 showing a statistically significant alteration, Table 4). Furthermore, a liver HCC study (TCGA, Provisional), which carried the largest sample size, was selected to analyze the screened proteins in cBioPortal databases. The results showed that $156(35 \%)$ patients/samples carried an alteration in at least one of the five genes queried using OncoPrint; the frequency of alteration in each gene is shown in Figure 2G. Most gene alterations included mutations and deletions. Most of the TP53 alterations were classified into missense mutations, truncating mutations, and deep deletions. Gene changes associated with RB1 included truncating mutations and deep deletions, whereas for TGFB1, GSTP1 and GSTM1 amplifications were the most common gene alterations.

Curcumin-associated genes and survival in HCC

Peer] reviewing PDF | (2021:05:61606:2:0:NEW 21 Sep 2021) 
279 Kaplan-Meier analysis was used to perform survival analysis of patients with HCC based on the 280 five selected genes (TP53, RB1, TGFB1, GSTP1 and GSTM1). A total of 364 patients were 281 involved in this analysis for OS (Menyhárt et al. 2018). As shown in Figure 3B-F, high mRNA 282 levels of TP53, RB1, and GSTM1 indicated increased OS in HCC, whereas elevated mRNA levels

283

284

285

286

287

288

289

290

291

292

293

294

295

296

297

298

299

300

301

302

303

304

305

306

307

308

309

310

311

312

313

314

315

316

317

318

of TGFB1 were correlated with poor prognosis in the same group of patients.

Curcumin-induced decrease in the activity of HepG2 and Hep3B cells

The test results showed that cell viability appeared dependent on the concentration of curcumin. For Hep3B cells, curcumin $(10 \mu \mathrm{M})$ significantly decreased cell viability after 48 and $72 \mathrm{~h}$, and 20 and $30 \mu \mathrm{M}$ of curcumin were found to significantly reduce cell viability after 24,48 , and $72 \mathrm{~h}$ (Figure 4A). For HepG2 cells, curcumin $(20$ and $30 \mu \mathrm{M})$ significantly decreased the cell viability after 48 and $72 \mathrm{~h}$ (Figure 4B). According to these results, the concentration of curcumin was chosen to be $20 \mu \mathrm{M}$ and the duration of treatment was chosen to be $48 \mathrm{~h}$ for subsequent testing.

Curcumin regulation of TGFB1 and GSTP1 expression in Hep3B cells The expression of TP53, RB1, TGFB1, GSTP1, and GSTM1 was assessed using qPCR in Hep3B cells treated with $20 \mu \mathrm{M}$ curcumin for $48 \mathrm{~h}$. The results showed that $20 \mu \mathrm{M}$ curcumin regulated the expression of TGFB1 and GSTP1 in Hep3B cells, but it had no significant effect on the expression of other genes (Figure 4C and 4D). After treatment of Hep3B cells with 0 and $20 \mu \mathrm{M}$ curcumin for $48 \mathrm{~h}$, the levels of TGFB1 and GSTP1 protein were detected via western blot. Because the protein background expression of GSTP1 was low, the expression of GSTP1 at the protein level failed to be detected. The results showed that $20 \mu \mathrm{M}$ curcumin did not regulate the expression of TGFB1 in HepG2 cells (Figure 4E, 4F), but moderately inhibited the expression of TGFB1 in Hep3B cells (Figure 4G, 4H).

\section{Discussion}

Over the past several years, numerous studies have evaluated the effects of curcumin and its analogs in diverse cancers in vitro and in vivo. According to these studies, the potential use of curcumin as a chemopreventive and therapeutic agent in cancers depends on its potent antioxidant and anti-inflammatory activities as well as its ability to modulate various molecular signaling mechanisms (Gupta et al. 2013; Hatcher et al. 2008; Huminiecki et al. 2017; Prasad et al. 2014). Nevertheless, the mechanism underlying the wide range of anti-cancer effects of curcumin remains incomplete. Current knowledge about curcumin's functions and mechanisms is based on conventional experiments, which have yet to be fully integrated and understood. Therefore, new analytical methods are needed to correlate curcumin with its target proteins and the observed biological effects. Functional/activity network (FAN)(Hsieh et al. 2016) is a new analytical method, which elucidates the molecular mechanisms of a drug and its association with clinical outcomes in cancer by using a set of web-based tools, such as DrugBank, STRING, cBioPortal, CTD, or Cytoscape. Using this method, a functional drug-target-cancer network analysis of gene-

Peer) reviewing PDF | (2021:05:61606:2:0:NEW 21 Sep 2021) 
319 phenotype connectivity associated with curcumin was conducted in this study. This study bridged 320 curcumin with its primary or secondary targets and illustrated the underlying mechanisms of 321 curcumin and its clinical outcomes in HCC.

322 The effects of curcumin and its analogs have been a subject of investigation over the past decade 323 in preclinical models of HCC (Darvesh et al. 2012), but research in this field is far from complete. 324 A search of PubMed using "curcumin" as the keyword returned more than 13000 publications,

325

326

327

328

329

330

331

332

333

334

335

336

337

338

339

340

341

342

343

344

345

346

347

348

349

350

351

352

353

354

355

356

357

358 whereas the phrase "curcumin and hepatocellular carcinoma" returned only 192 publications. By using FAN, more direct mechanisms may be mined with reasonable experimental feasibility to validate hypotheses explaining the effects of curcumin in HCC.

In this study, the feasibility of FAN analysis for identifying the underlying connectivity between curcumin and HCC was demonstrated.A network including five genes (TP53, RB1, TGFB1, GSTP1, and GSTM1) as targets of curcumin in HCC was identified via a functional drug-targetcancer network analysis. Among the five genes, TP53 and RB1 are tumor suppressor genes, which is consistent with the finding in this study that their high transcriptional expression is correlated with better OS in HCC. Similar to other cancers, TP53 and RB1 are the most commonly inactivated or mutated genes in the case of HCC. Moreover, mutual exclusivity analysis revealed a tendency toward concurrence between TP53 and RB1. Previous studies indicated that curcumin may inhibit cancer growth and induce apoptosis in colon cancer cells (Dasiram et al. 2017), ameliorate the in vitro efficacy of carfilzomib in human multiple myeloma cells (Allegra et al. 2018), promote apoptosis in non-small cell lung cancer (Ye et al. 2015), and inhibit cell growth in nasopharyngeal carcinoma mediated via the TP53 signaling pathway(Wu et al. 2014). Debata et al. reported that the sunitinib-curcumin combination was effective in restoring the tumor suppressor activity of the RB gene in renal cancer cells (Debata et al. 2013). Furthermore, Su et al. reported that curcumin significantly enhanced p53 or markedly inhibited the RB pathway by suppressing RB phosphorylation in the signaling pathways of glioblastoma ( $\mathrm{Su}$ et al. 2010). These studies demonstrated the anti-tumor activity of curcumin and prompted further investigation of curcumin in HCC.

In the case of HCC, most of the genetic alterations in TGFB1, GSTP1, and GSTM1 were amplifications, which may cause increased expression. However, the prognostic significance varied in this study. The amplification of TGFB1 indicated a worse OS in HCC, whereas amplifications of GSTM1 and GSTP1 (not statistically significant) indicated better prognosis. TGFB1 is a pleiotropic gene with a dual role in hepatocarcinogenesis: apoptosis induction in early phases, but promotion of tumorigenesis in cells with mechanisms to overcome the suppressor effects (Moreno-Caceres et al. 2017). Recent studies found that the expression of TGFB1 genes were downregulated in breast cancer cells treated with curcumin (Calaf \& Roy 2017). Glutathione S-transferases (GSTs) are a family of phase II detoxification enzymes that catalyze the conjugation of a wide variety of endogenous and exogenous toxins. A previous study showed that interindividual GST variation plays a central role in reducing cell exposure to carcinogens (Di Pietro et al. 2010). For example, reduced GSTP1 expression may contribute to oxidative stress in HCC (Li et al. 2013). Similar results were observed in breast cancer cells and curcumin-activated GSTP1 
359

360

361

362

363

364

365

366

367

368

369

370

371

372

373

374

375

376

377

378

379

380

381

382

383

384

385

386

387

388

389

390

391

392

393

394

395

396

397

398

expression via antioxidant response element (Nishinaka et al. 2007). However, studies linking curcumin and GST genes in HCC are rare, underscoring the need for further investigation in this field.

These results were partially confirmed by in vitro experiments. HepG2 and Hep3B were used as the experimental objects because of their advantages of fast growth and easy passage. The present study confirmed the inhibitory effect of curcumin on liver cancer cells, and this inhibition increased with time and concentration. In the liver HCC study (TCGA, Provisional), three genes (TGFB1, GSTP1, and GSTM1) showed amplification. For this reason, changes in these amplified genes were more easily detected using PCR. This was confirmed in the present study when it was found that two genes prone to amplification were suppressed in HCC cells treated with curcumin. The other genes, however, did not show changes in expression because the main means of mutation was not amplification mutations but truncating mutations and deep deletions. In terms of the relationship between curcumin and protein expression, only one protein change TGFB1 was detected by western blot. This may be because of the complexity of post-transcriptional regulation of mRNA, meaning that mRNA amplification may not be consistent with protein expression. The results of this study showed that TGFB1 expression was inconsistent at mRNA and protein levels after curcumin treatment, possibly because TGFB1 may be regulated by intracellular activators. In addition, although mRNA concentration is widely used as a surrogate for protein abundance, studies comparing mRNA and protein expression on a global scale indicate that mRNA levels only partly correlate with the corresponding protein concentrations. It has been estimated that protein concentrations are determined by the corresponding mRNA concentrations by only $20 \%-40 \%$ (Nie et al. 2006; Tian et al. 2004).

Furthermore, studies have proved that TGFB1 has a dual role in the development and progression of HCC. In the previous analysis, elevated mRNA levels of TGFB1 were correlated with poor prognosis in HCC patients. This may be because TGFB1 promoted the growth, invasion, and metastasis of HCC cells through the epithelial-mesenchymal transition (Bierie \& Moses 2006; Massagué 2008; Matsuura et al.). Regarding HCC cell levels, TGFB1 was a potent growth inhibitor and induces apoptosis in these cells, so it is regarded as a tumor-suppressive cytokine (Senturk et al. 2010). Therefore, further studies are needed to validate and establish the specific molecular mechanisms by which curcumin regulates HCC cells.

\section{Conclusions}

In summary, by using DrugBank, STRING, CDC, and cBioPortal databases, the connectivity between curcumin and $\mathrm{HCC}$ was discovered. Curcumin has the potential to become an alternative chemotherapy or chemoprevention treatment for HCC. The drug-target-cancer network analysis utilized in this study facilitated the testing and validation of reasonable hypotheses explaining curcumin-induced gene alterations in cancers by applying the available biological information in 
399 studies from bedside to bench. As advances in curcumin research using traditional experimental 400 approaches continue, additional drug targets will undoubtedly be identified, leading to improved 401 curcumin-related genetic networks, signaling pathways, and cancer types.

402

403

404 List of abbreviations

405

406 DPTs: direct protein targets

407 IPTs: indirect protein targets

408 PPI: protein-protein interaction

409 KEGG: Kyoto Encyclopedia of Genes and Genomes

410 CTD: Comparative Toxicogenomics Database

411 HCC: hepatocellular carcinoma

412 cBioPortal: cBio Cancer Genomics Portal

413 OS: overall survival

414 FAN: functional/activity network

415 OD: optical density

416 MTT: (3-(4, 5)-dimethylthiahiazo (-z-y1)-3, 5-di-phenytetrazoliumromide)

417

418

Acknowledgments

419

420

We would like to thank LetPub (www.letpub.com) for its linguistic assistance during the 421 preparation of this manuscript.

\section{References}

Ahn SM, Jang SJ, Shim JH, Kim D, Hong SM, Sung CO, Baek D, Haq F, Ansari AA, Lee SY, Chun SM, Choi S, Choi HJ, Kim J, Kim S, Hwang S, Lee YJ, Lee JE, Jung WR, Jang HY, Yang E, Sung WK, Lee NP, Mao M, Lee C, ZucmanRossi J, Yu E, Lee HC, and Kong G. 2014. Genomic portrait of resectable hepatocellular carcinomas: 
435

436

437

438

439

440

441

442

443

444

445

446

447

448

449

450

451

452

453

454

455

456

457

458

459

460

461

462

463

464

465

466

467

468

469

470

471

472

473

474

475

476

477

478

479

480

481

482

483

484

485

486

implications of RB1 and FGF19 aberrations for patient stratification. Hepatology 60:1972-1982. 10.1002/hep. 27198

Allegra A, Speciale A, Molonia MS, Guglielmo L, Musolino C, Ferlazzo G, Costa G, Saija A, and Cimino F. 2018. Curcumin ameliorates the in vitro efficacy of carfilzomib in human multiple myeloma U266 cells targeting p53 and NF-kappaB pathways. Toxicol In Vitro 47:186-194. 10.1016/j.tiv.2017.12.001

Banerjee S, Ji C, Mayfield JE, Goel A, Xiao J, Dixon JE, and Guo X. 2018. Ancient drug curcumin impedes $26 \mathrm{~S}$ proteasome activity by direct inhibition of dual-specificity tyrosine-regulated kinase 2. Proc Natl Acad Sci U S A 115:8155-8160. 10.1073/pnas.1806797115

Bianchi G, Ravera S, Traverso C, Amaro A, Piaggio F, Emionite L, Bachetti T, Pfeffer U, and Raffaghello L. 2018. Curcumin induces a fatal energetic impairment in tumor cells in vitro and in vivo by inhibiting ATPsynthase activity. Carcinogenesis 39:1141-1150. 10.1093/carcin/bgy076

Bierie B, and Moses HL. 2006. TGF beta: the molecular Jekyll and Hyde of cancer. Nature reviews Cancer 6:506-520. Calaf GM, and Roy D. 2017. Metastatic genes targeted by an antioxidant in an established radiation- and estrogenbreast cancer model. Int J Oncol 51:1590-1600. 10.3892/ijo.2017.4125

Cerami E, Gao J, Dogrusoz U, Gross BE, Sumer SO, Aksoy BA, Jacobsen A, Byrne CJ, Heuer ML, Larsson E, Antipin Y, Reva B, Goldberg AP, Sander C, and Schultz N. 2012. The cBio cancer genomics portal: an open platform for exploring multidimensional cancer genomics data. Cancer Discov 2:401-404. 10.1158/2159-8290.CD12-0095

Chuang SE, Kuo ML, Hsu CH, Chen CR, Lin JK, Lai GM, Hsieh CY, and Cheng AL. 2000. Curcumin-containing diet inhibits diethylnitrosamine-induced murine hepatocarcinogenesis. Carcinogenesis 21:331-335. 10.1093/carcin/21.2.331

Darvesh AS, Aggarwal BB, and Bishayee A. 2012. Curcumin and liver cancer: a review. Curr Pharm Biotechnol 13:218-228.

Dasiram JD, Ganesan R, Kannan J, Kotteeswaran V, and Sivalingam N. 2017. Curcumin inhibits growth potential by G1 cell cycle arrest and induces apoptosis in p53-mutated COLO 320DM human colon adenocarcinoma cells. Biomed Pharmacother 86:373-380. 10.1016/j.biopha.2016.12.034

Davis AP, Grondin CJ, Johnson RJ, Sciaky D, McMorran R, Wiegers J, Wiegers TC, and Mattingly CJ. 2019. The Comparative Toxicogenomics Database: update 2019. Nucleic Acids Res 47:D948-D954. 10.1093/nar/gky868

Debata PR, Begum S, Mata A, Genzer O, Kleiner MJ, Banerjee P, and Castellanos MR. 2013. Curcumin potentiates the ability of sunitinib to eliminate the VHL-lacking renal cancer cells 786-O: rapid inhibition of $\mathrm{Rb}$ phosphorylation as a preamble to cyclin D1 inhibition. Anticancer Agents Med Chem 13:1508-1513.

Di Pietro G, Magno LA, and Rios-Santos F. 2010. Glutathione S-transferases: an overview in cancer research. Expert Opin Drug Metab Toxicol 6:153-170. 10.1517/17425250903427980

Ferlay J, Soerjomataram I, Dikshit R, Eser S, Mathers C, Rebelo M, Parkin DM, Forman D, and Bray F. 2015. Cancer incidence and mortality worldwide: sources, methods and major patterns in GLOBOCAN 2012. Int J Cancer 136:E359-386. 10.1002/ijc.29210

Fu S, Zhang Y, Shi J, Hao D, and Zhang P. 2019. Identification of gene-phenotype connectivity associated with flavanone naringenin by functional network analysis. PeerJ 7:e6611. 10.7717/peerj.6611

Fujimoto A, Totoki Y, Abe T, Boroevich KA, Hosoda F, Nguyen HH, Aoki M, Hosono N, Kubo M, Miya F, Arai Y, Takahashi H, Shirakihara T, Nagasaki M, Shibuya T, Nakano K, Watanabe-Makino K, Tanaka H, Nakamura H, Kusuda J, Ojima H, Shimada K, Okusaka T, Ueno M, Shigekawa Y, Kawakami Y, Arihiro K, Ohdan H, Gotoh K, Ishikawa O, Ariizumi S, Yamamoto M, Yamada T, Chayama K, Kosuge T, Yamaue H, Kamatani N, Miyano S, Nakagama H, Nakamura Y, Tsunoda T, Shibata T, and Nakagawa H. 2012. Whole-genome sequencing of liver cancers identifies etiological influences on mutation patterns and recurrent mutations in chromatin regulators. Nat Genet 44:760-764. 10.1038/ng.2291

Gao J, Aksoy BA, Dogrusoz U, Dresdner G, Gross B, Sumer SO, Sun Y, Jacobsen A, Sinha R, Larsson E, Cerami E, Sander C, and Schultz N. 2013. Integrative analysis of complex cancer genomics and clinical profiles using the cBioPortal. Sci Signal 6:pl1. 10.1126/scisignal.2004088

Ghasemi F, Shafiee M, Banikazemi Z, Pourhanifeh MH, Khanbabaei H, Shamshirian A, Amiri Moghadam S, ArefNezhad R, Sahebkar A, Avan A, and Mirzaei H. 2019. Curcumin inhibits NF-kB and Wnt/ $\beta$-catenin pathways in cervical cancer cells. Pathol Res Pract 215:152556. 10.1016/j.prp.2019.152556

Peer) reviewing PDF | (2021:05:61606:2:0:NEW 21 Sep 2021) 
487

488

489

490

491

492

493

494

495

496

497

498

499

500

501

502

503

504

505

506

507

508

509

510

511

512

513

514

515

516

517

518

519

520

521

522

523

524

525

526

527

528

529

530

531

532

533

534

535

536

537

538

539

Gupta SC, Patchva S, and Aggarwal BB. 2013. Therapeutic roles of curcumin: lessons learned from clinical trials. Aaps j 15:195-218. 10.1208/s12248-012-9432-8

Harding JJ, Nandakumar S, Armenia J, Khalil DN, Albano M, Ly M, Shia J, Hechtman JF, Kundra R, El Dika I, Do RK, Sun Y, Kingham TP, D'Angelica MI, Berger MF, Hyman DM, Jarnagin W, Klimstra DS, Janjigian YY, Solit DB, Schultz N, and Abou-Alfa GK. 2019. Prospective Genotyping of Hepatocellular Carcinoma: Clinical Implications of Next-Generation Sequencing for Matching Patients to Targeted and Immune Therapies. Clin Cancer Res 25:2116-2126. 10.1158/1078-0432.CCR-18-2293

Hatcher H, Planalp R, Cho J, Torti FM, and Torti SV. 2008. Curcumin: from ancient medicine to current clinical trials. Cell Mol Life Sci 65:1631-1652. 10.1007/s00018-008-7452-4

Hsieh TC, Wu ST, Bennett DJ, Doonan BB, Wu E, and Wu JM. 2016. Functional/activity network (FAN) analysis of gene-phenotype connectivity liaised by grape polyphenol resveratrol. Oncotarget 7:38670-38680. 10.18632/oncotarget.9578

Huang MT, Lou YR, Ma W, Newmark HL, Reuhl KR, and Conney AH. 1994. Inhibitory effects of dietary curcumin on forestomach, duodenal, and colon carcinogenesis in mice. Cancer Res 54:5841-5847.

Huminiecki L, Horbanczuk J, and Atanasov AG. 2017. The functional genomic studies of curcumin. Semin Cancer Biol 46:107-118. 10.1016/j.semcancer.2017.04.002

Kawamori T, Lubet R, Steele VE, Kelloff GJ, Kaskey RB, Rao CV, and Reddy BS. 1999. Chemopreventive effect of curcumin, a naturally occurring anti-inflammatory agent, during the promotion/progression stages of colon cancer. Cancer Res 59:597-601.

Lan K, Wang DT, Fong S, Liu LS, Wong KKL, and Dey N. 2018. A Survey of Data Mining and Deep Learning in Bioinformatics. J Med Syst 42:139. 10.1007/s10916-018-1003-9

Li T, Zhao XP, Wang LY, Gao S, Zhao J, Fan YC, and Wang K. 2013. Glutathione S-transferase P1 correlated with oxidative stress in hepatocellular carcinoma. Int J Med Sci 10:683-690. 10.7150/ijms.5947

Massagué J. 2008. TGF $\beta$ in Cancer. Cell 134:215-230.

Matsuura I, Denissova NG, Wang G, He D, Long J, and Liu F. Cyclin-dependent kinases regulate the antiproliferative function of Smads. Nature.

McGlynn KA, Petrick JL, and London WT. 2015. Global epidemiology of hepatocellular carcinoma: an emphasis on demographic and regional variability. Clin Liver Dis 19:223-238. 10.1016/j.cld.2015.01.001

Menyhárt O, Nagy Á, and Győrffy B. 2018. Determining consistent prognostic biomarkers of overall survival and vascular invasion in hepatocellular carcinoma. $R$ Soc Open Sci 5:181006. 10.1098/rsos.181006

Moreno-Caceres J, Caballero-Diaz D, Nwosu ZC, Meyer C, Lopez-Luque J, Malfettone A, Lastra R, Serrano T, Ramos E, Dooley S, and Fabregat I. 2017. The level of caveolin-1 expression determines response to TGF-beta as a tumour suppressor in hepatocellular carcinoma cells. Cell Death Dis 8:e3098. 10.1038/cddis.2017.469

Nagy A, Lanczky A, Menyhart O, and Gyorffy B. 2018. Validation of miRNA prognostic power in hepatocellular carcinoma using expression data of independent datasets. Sci Rep 8:9227. 10.1038/s41598-018-27521-y

Nie L, Wu G, and Zhang W. 2006. Correlation between mRNA and protein abundance in Desulfovibrio vulgaris: a multiple regression to identify sources of variations. Biochem Biophys Res Commun 339:603-610. 10.1016/j.bbrc.2005.11.055

Nishinaka T, Ichijo Y, Ito M, Kimura M, Katsuyama M, Iwata K, Miura T, Terada T, and Yabe-Nishimura C. 2007. Curcumin activates human glutathione S-transferase P1 expression through antioxidant response element. Toxicol Lett 170:238-247. 10.1016/j.toxlet.2007.03.011

Pilati C, Letouze E, Nault JC, Imbeaud S, Boulai A, Calderaro J, Poussin K, Franconi A, Couchy G, Morcrette G, Mallet M, Taouji S, Balabaud C, Terris B, Canal F, Paradis V, Scoazec JY, de Muret A, Guettier C, Bioulac-Sage P, Chevet E, Calvo F, and Zucman-Rossi J. 2014. Genomic profiling of hepatocellular adenomas reveals recurrent FRK-activating mutations and the mechanisms of malignant transformation. Cancer Cell 25:428441. 10.1016/j.ccr.2014.03.005

Prasad S, Gupta SC, Tyagi AK, and Aggarwal BB. 2014. Curcumin, a component of golden spice: from bedside to bench and back. Biotechnol Adv 32:1053-1064. 10.1016/j.biotechadv.2014.04.004

Schulze K, Imbeaud S, Letouze E, Alexandrov LB, Calderaro J, Rebouissou S, Couchy G, Meiller C, Shinde J, Soysouvanh F, Calatayud AL, Pinyol R, Pelletier L, Balabaud C, Laurent A, Blanc JF, Mazzaferro V, Calvo F, Villanueva A, Nault JC, Bioulac-Sage P, Stratton MR, Llovet JM, and Zucman-Rossi J. 2015. Exome sequencing of hepatocellular carcinomas identifies new mutational signatures and potential therapeutic targets. Nat Genet 47:505-511. 10.1038/ng.3252

Peer) reviewing PDF | (2021:05:61606:2:0:NEW 21 Sep 2021) 
540

541

542

543

544

545

546

547

548

549

550

551

552

553

554

555

556

557

558

559

560

561

562

563

564

565

566

567

568

569

570

571

572

573

574

575

576

577

578

579

580
Senturk S, Mumcuoglu M, Gursoy ${ }^{\text {TEL }}$ uzugullu O, Cingoz B, Akcali KC, and Ozturk M. 2010. Transforming growth factor - beta induces senescence in hepatocellular carcinoma cells and inhibits tumor growth. Hepatology 52.

Shannon P, Markiel A, Ozier O, Baliga NS, Wang JT, Ramage D, Amin N, Schwikowski B, and Ideker T. 2003. Cytoscape: a software environment for integrated models of biomolecular interaction networks. Genome Res 13:2498-2504. 10.1101/gr.1239303

Su CC, Wang MJ, and Chiu TL. 2010. The anti-cancer efficacy of curcumin scrutinized through core signaling pathways in glioblastoma. Int J Mol Med 26:217-224. 10.3892/ijmm_00000455

Su G, Morris JH, Demchak B, and Bader GD. 2014. Biological network exploration with Cytoscape 3. Curr Protoc Bioinformatics 47:8 13 11-24. 10.1002/0471250953.bi0813s47

Szklarczyk D, Franceschini A, Wyder S, Forslund K, Heller D, Huerta-Cepas J, Simonovic M, Roth A, Santos A, Tsafou KP, Kuhn M, Bork P, Jensen LJ, and von Mering C. 2015. STRING v10: protein-protein interaction networks, integrated over the tree of life. Nucleic Acids Res 43:D447-452. 10.1093/nar/gku1003

Szklarczyk D, Gable AL, Lyon D, Junge A, Wyder S, Huerta-Cepas J, Simonovic M, Doncheva NT, Morris JH, Bork P, Jensen $L$, and Mering CV. 2019. STRING v11: protein-protein association networks with increased coverage, supporting functional discovery in genome-wide experimental datasets. Nucleic Acids Res 47:D607-D613. 10.1093/nar/gky1131

Tian Q, Stepaniants S, Mao M, Weng L, Feetham M, Doyle M, Yi E, Dai H, Thorsson V, Eng J, Goodlett D, Berger J, Gunter B, Linseley P, Stoughton R, Aebersold R, Collins S, Hanlon W, and Hood L. 2004. Integrated Genomic and Proteomic Analyses of Gene Expression in Mammalian Cells. Molecular \& cellular proteomics : MCP 3:960-969. 10.1074/mcp.M400055-MCP200

Wishart DS, Feunang YD, Guo AC, Lo EJ, Marcu A, Grant JR, Sajed T, Johnson D, Li C, Sayeeda Z, Assempour N, Iynkkaran I, Liu Y, Maciejewski A, Gale N, Wilson A, Chin L, Cummings R, Le D, Pon A, Knox C, and Wilson M. 2018. DrugBank 5.0: a major update to the DrugBank database for 2018. Nucleic Acids Res 46:D1074D1082. 10.1093/nar/gkx1037

Wishart DS, Knox C, Guo AC, Cheng D, Shrivastava S, Tzur D, Gautam B, and Hassanali M. 2008. DrugBank: a knowledgebase for drugs, drug actions and drug targets. Nucleic Acids Res 36:D901-906. 10.1093/nar/gkm958

Wu J, Tang Q, Zhao S, Zheng F, Wu Y, Tang G, and Hahn SS. 2014. Extracellular signal-regulated kinase signalingmediated induction and interaction of FOXO3a and p53 contribute to the inhibition of nasopharyngeal carcinoma cell growth by curcumin. Int J Oncol 45:95-103. 10.3892/ijo.2014.2420

Ye M, Zhang J, Zhang J, Miao Q, Yao L, and Zhang J. 2015. Curcumin promotes apoptosis by activating the p53-miR192-5p/215-XIAP pathway in non-small cell lung cancer. Cancer Lett 357:196-205. 10.1016/j.canlet.2014.11.028

Zhang C, Yang F, Ni S, Teng W, and Ning Y. 2018. Drug-target-disease network analysis of gene-phenotype connectivity for genistein in ovarian cancer. Onco Targets Ther 11:8901-8908. 10.2147/OTT.S183302

Zheng J, Sadot E, Vigidal JA, Klimstra DS, Balachandran VP, Kingham TP, Allen PJ, D'Angelica MI, DeMatteo RP, Jarnagin WR, and Ventura A. 2018. Characterization of hepatocellular adenoma and carcinoma using microRNA profiling and targeted gene sequencing. PLoS One 13:e0200776.

10.1371/journal.pone.0200776 
Figure 1

Figure 1

Workflow chart of study. 


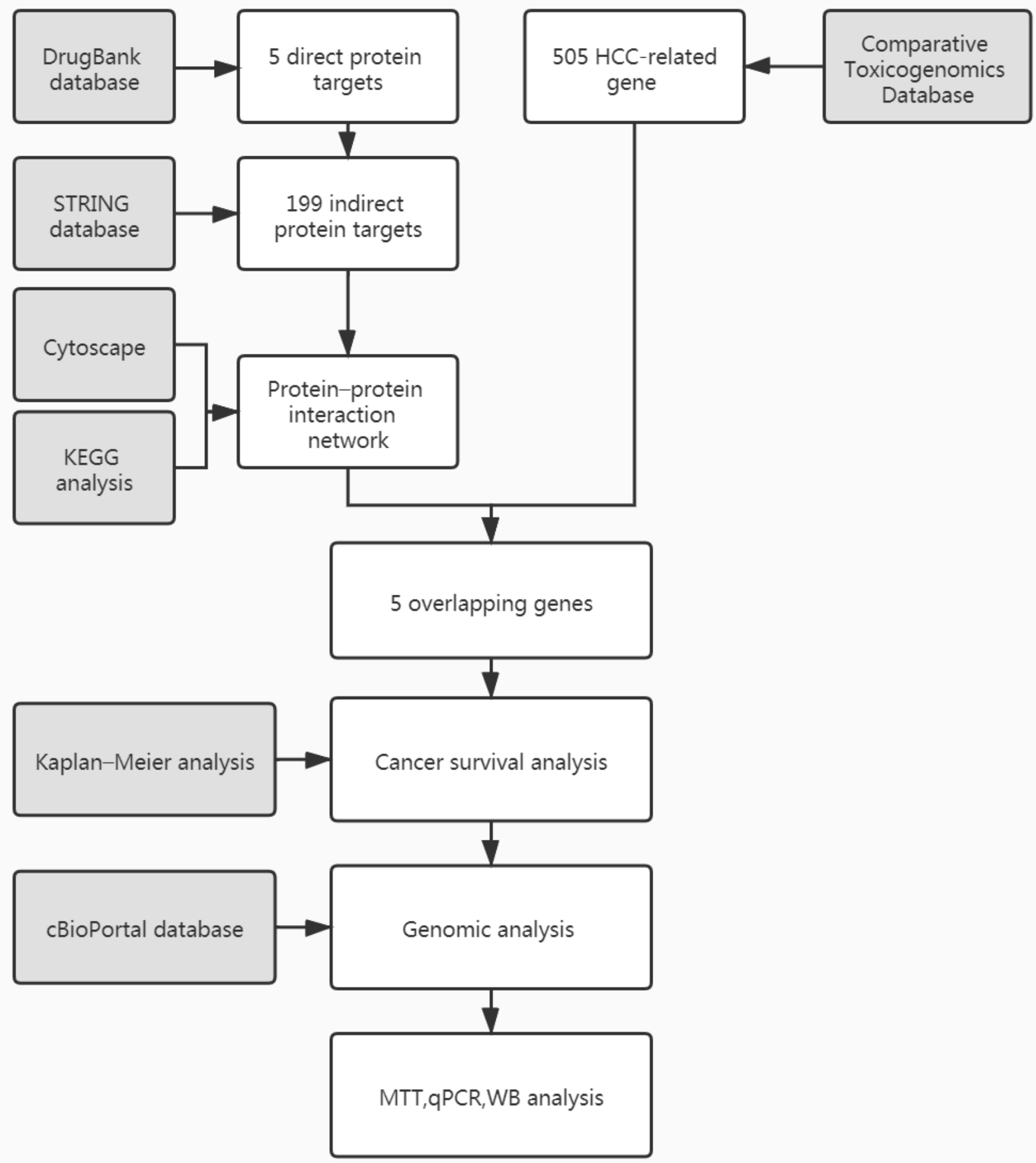




\section{Figure 2}

Figure 2

(A) The structure of curcumin. (B) The interactions of 5 DPTs of curcumin by STRING with a minimum required interaction score 0.2.(C) PPI network of curcumin-mediated proteins analyzed by Cytoscape. Network nodes represent proteins, and edges represent proteinprotein associations. High node degree values are represented by big sizes. (D) The top 15 statistically enriched KEGG pathways and involved gene numbers.(E) Venn diagrams for intersections of 3 gene sets. (F) A visual display of the network connected to 5 selected protein targets (TP53, RB1, TGFB1, GSTP1, and GSTM1). Neighboring proteins connected to the 5 query proteins, filtered from no more than 5 interactors to no interactor.(G) Overview of genetic alterations related to curcumin-associated genes in genomics data sets available in 7 different HCC studies in cBioPortal databases. 
A

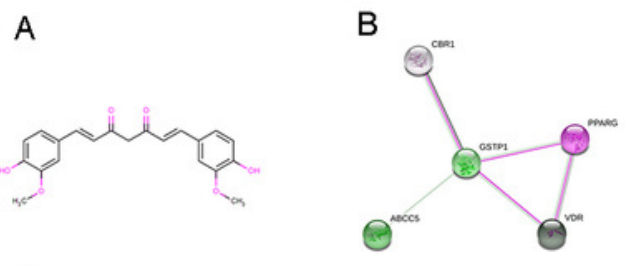

C

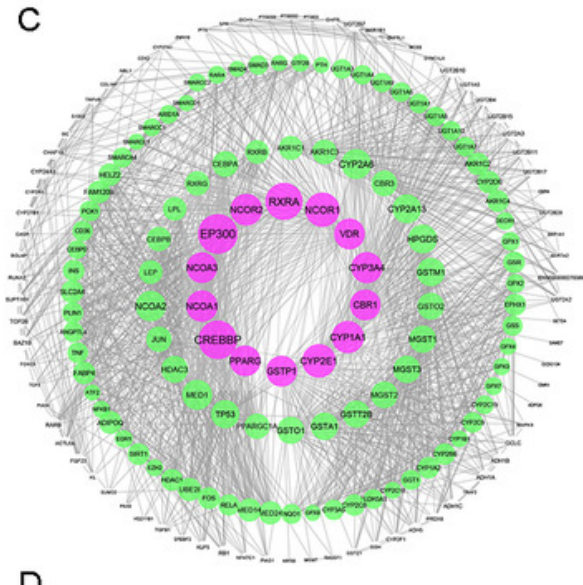

D

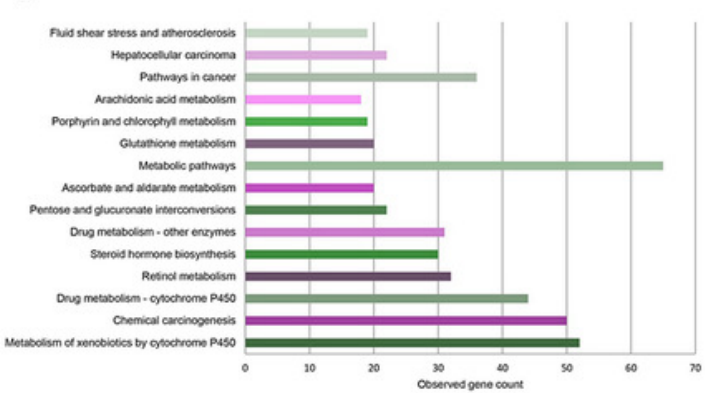

E
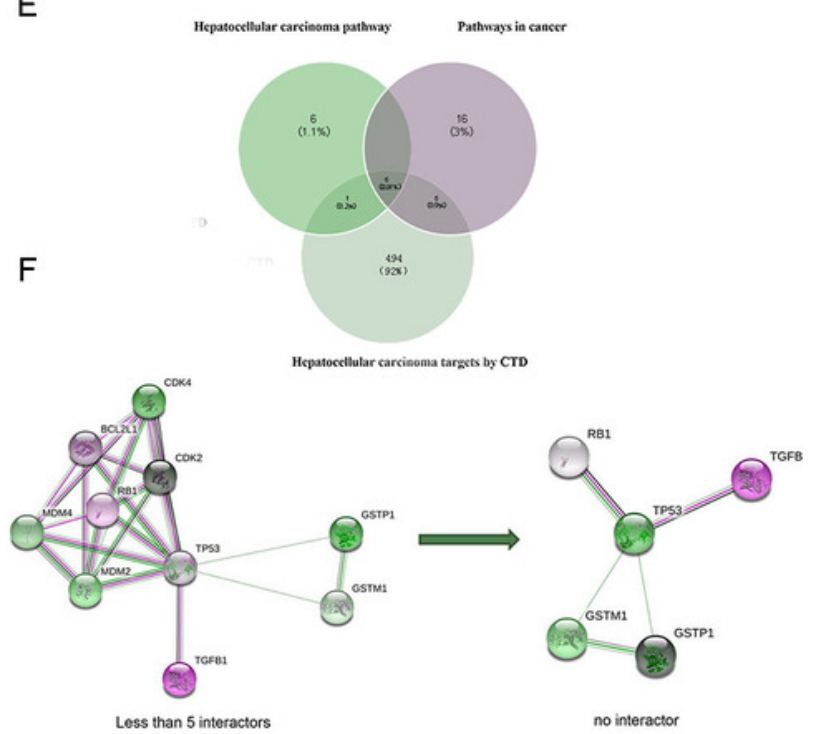

$\mathrm{F}$

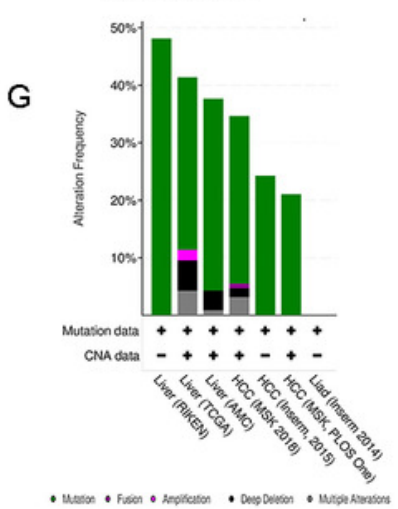




\section{Figure 3}

Figure 3

(A)A visual heatmap of mRNA-level alterations based on 5 genes (TP53, RB1, TGFB1, GSTP1, and GSTM1) across a HCC study (data taken from the Liver HCC (TCGA, Provisional) study) in cBioPortal databases. Each row represents a gene, and each column represents a tumor sample.Survival analysis of 5 selected genes according to mRNA expression in HCC: (B) TP53, (C) RB1, (D) GSTM1, (E) GSTP1, and (F) TGFB1. Magenta lines indicate high levels of mRNA expression, while black lines indicate low levels of mRNA expression.

A

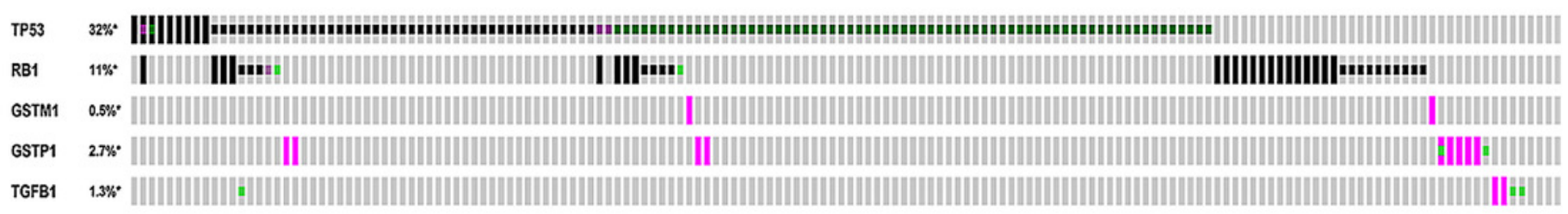
Genetic Alteration

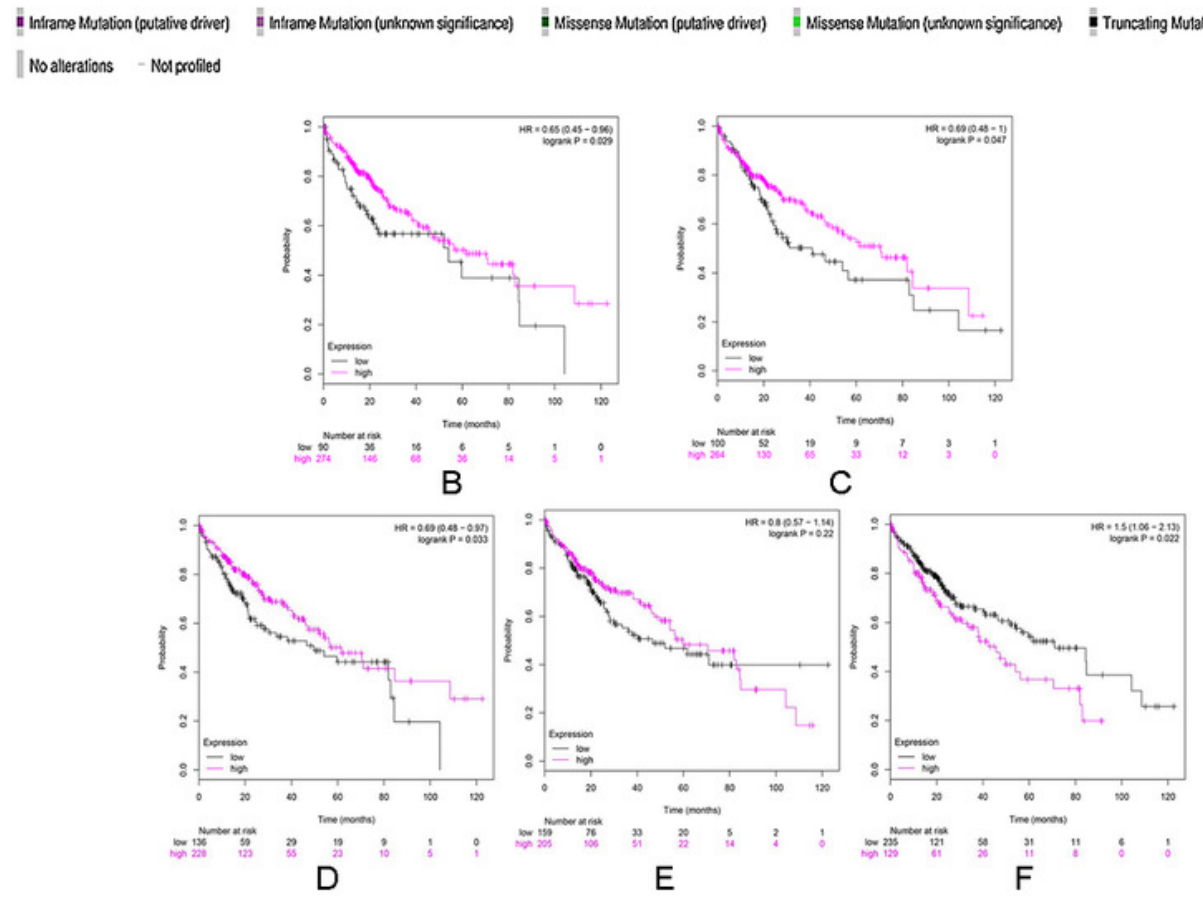




\section{Figure 4}

Figure 4

Validation experiment in vitro (A) Cell viability inhibition experiment of HepG2. (B) Cell viability inhibition experiment of Hep3B. (C) qPCR analysis of five overlapping genes in HepG2. (D) qPCR analysis of five overlapping genes in Hep3B.(E)Western blot analysis of TGFB1 in HepG2. (F) Quantitative analysis of TGFB1 protein expression in HepG2.(E)Western blot analysis of TGFB1 in Hep3B. (F) Quantitative analysis of TGFB1 protein expression in Hep3B. 

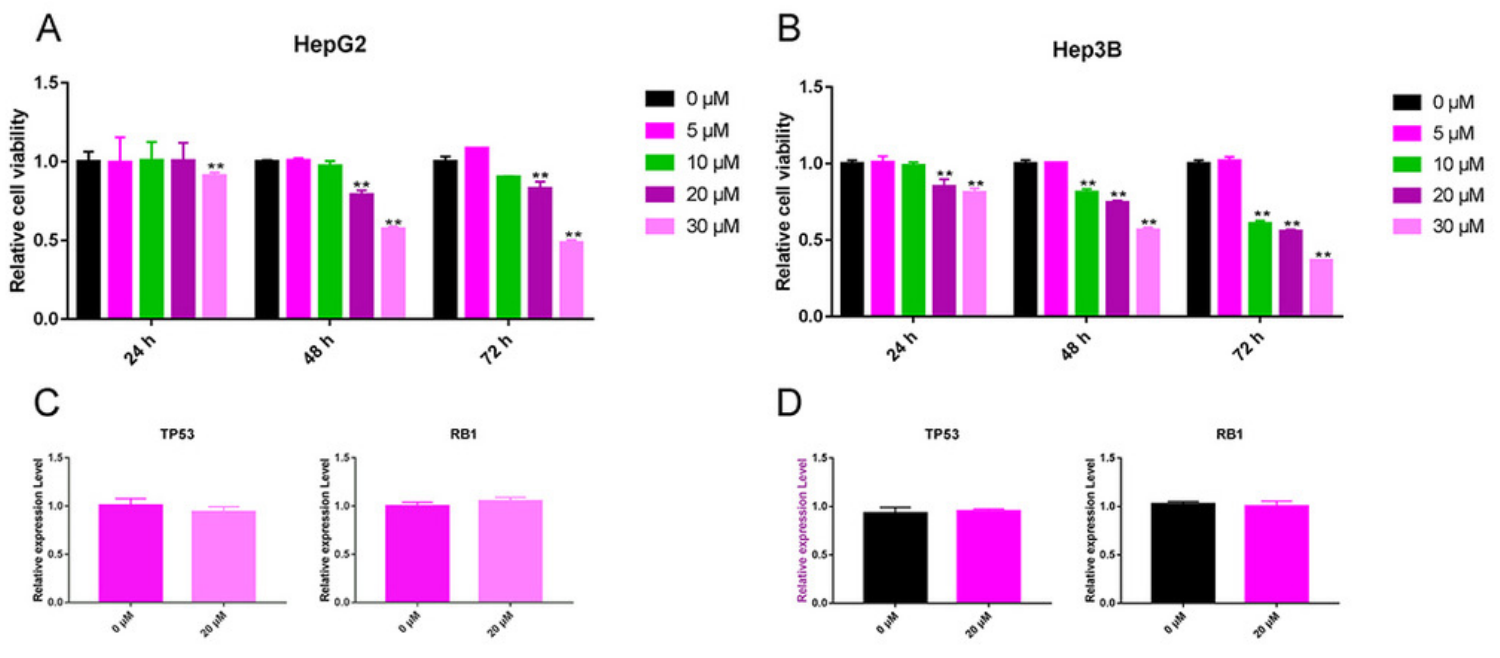

$\mathrm{D}$
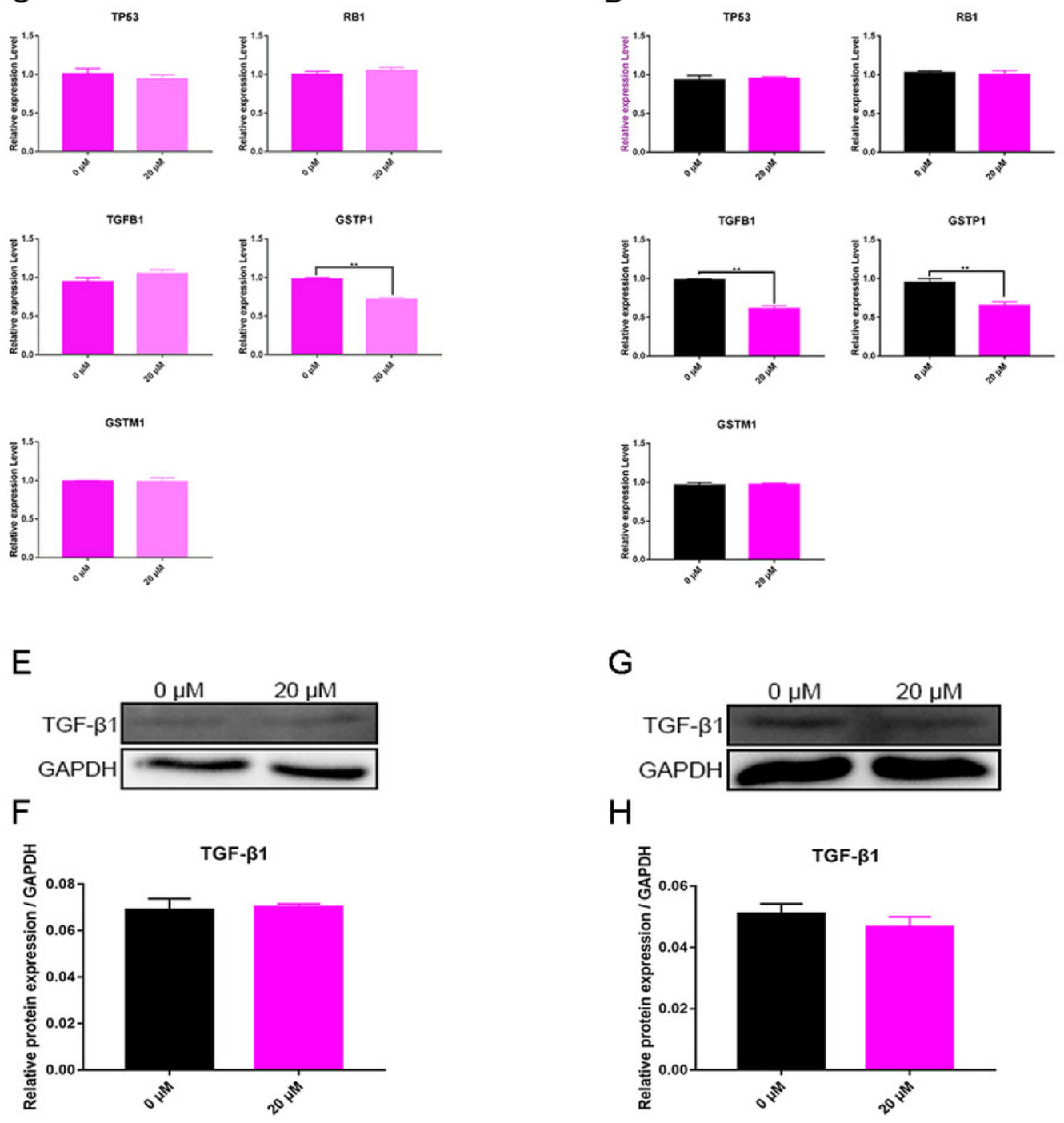

$\mathrm{H}$

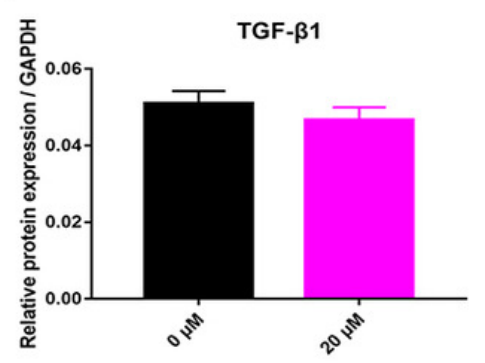




\section{Table $\mathbf{1}$ (on next page)}

Table 1

Direct protein targets of curcumin identified using DrugBank 
1 Table 1. Direct protein targets of curcumin identified using DrugBank

\begin{tabular}{|c|c|c|c|}
\hline Uniprot & Gene & & \\
\hline ID & name & Targets & General function \\
\hline P37231 & PPARG & Peroxisome proliferator-activated receptor gamma & Zinc ion binding \\
\hline \multirow[t]{3}{*}{ P11473 } & VDR & Vitamin D3 receptor & Zinc ion binding \\
\hline & & & Organic anion \\
\hline & & & transmembrane \\
\hline \multirow[t]{3}{*}{ O15440 } & $\mathrm{ABCC} 5$ & Multidrug resistance-associated protein 5 & transporter activity \\
\hline & & & Prostaglandin-e2 \\
\hline & & & 9-reductase \\
\hline \multirow[t]{3}{*}{ P16152 } & CBR1 & Carbonyl reductase [NADPH] 1 & activity \\
\hline & & & S- \\
\hline & & & nitrosoglutathione \\
\hline P09211 & GSTP1 & Glutathione S-transferase P & binding \\
\hline
\end{tabular}

2

3

4

5 
Table 2 (on next page)

Table 2

Protein targets with degree value $\geq 50$ 
1 Table 2. Protein targets with degree value $\geq 50$

\begin{tabular}{llll}
\hline Gene & Node degree value & Gene & Node degree value \\
\hline EP300 & 75 & NCOA1 & 52 \\
CREBBP & 72 & CYP3A4 & 51 \\
RXRA & 68 & GSTP1 & 50 \\
NCOR1 & 60 & NCOA3 & 50 \\
CYP2E1 & 58 & VDR & 50 \\
CYP1A1 & 54 & CBR1 & 50 \\
NCOR2 & 53 & PPARG & 50 \\
\hline
\end{tabular}

2 
Table 3 (on next page)

Table 3

Top 15 enriched KEGG pathways identified using STRING 
1 Table 3. Top 15 enriched KEGG pathways identified using STRING

\begin{tabular}{|c|c|c|c|}
\hline Pathways & gene count & FDR & matching proteins \\
\hline Metabolism of & 52 & $5.68 \mathrm{E}-70$ & ADH1A,ADH1B,ADH1C,ADH5,AKR1C1,AKR7 \\
\hline xenobiotics by & & & A2,ALDH3A1,CBR1,CBR3,CYP1A1,CYP1A2,C \\
\hline cytochrome & & & YP1B1,CYP2A13,CYP2A6,CYP2B6,CYP2C9,C \\
\hline \multirow[t]{8}{*}{$\mathrm{P} 450$} & & & YP2D6,CYP2E1,CYP2F1,CYP3A4,CYP3A5,EN \\
\hline & & & SG00000270386,EPHX1,GSTA1,GSTM1,GSTO1 \\
\hline & & & ,GSTO2,GSTP1,GSTT2B,HPGDS,HSD11B1,MG \\
\hline & & & ST1,MGST2,MGST3,UGT1A1,UGT1A10,UGT1 \\
\hline & & & A3,UGT1A4,UGT1A5,UGT1A6,UGT1A7,UGT1 \\
\hline & & & A8,UGT1A9,UGT2A2,UGT2A3,UGT2B10,UGT \\
\hline & & & 2B11,UGT2B15,UGT2B17,UGT2B28,UGT2B4, \\
\hline & & & UGT2B7 \\
\hline Chemical & 50 & $1.80 \mathrm{E}-65$ & ADH1A,ADH1B,ADH1C,ADH5,AKR1C2,ALD \\
\hline \multirow[t]{6}{*}{ carcinogenesis } & & & H3A1,CBR1,CYP1A1,CYP1A2,CYP1B1,CYP2A \\
\hline & & & 13,CYP2A6,CYP2C18,CYP2C19,CYP2C8,CYP2 \\
\hline & & & C9,CYP2E1,CYP3A4,CYP3A5,ENSG000002703 \\
\hline & & & 86,EPHX1,GSTA1,GSTM1,GSTO1,GSTO2,GST \\
\hline & & & P1,GSTT2B,HPGDS,HSD11B1,MGST1,MGST2, \\
\hline & & & MGST3,UGT1A1,UGT1A10,UGT1A3,UGT1A4, \\
\hline
\end{tabular}




\begin{tabular}{|c|c|c|c|}
\hline & & & UGT1A5,UGT1A6,UGT1A7,UGT1A8,UGT1A9, \\
\hline & & & UGT2A2,UGT2A3,UGT2B10,UGT2B11,UGT2B \\
\hline & & & 15,UGT2B17,UGT2B28,UGT2B4,UGT2B7 \\
\hline Drug & 44 & $1.30 \mathrm{E}-57$ & ADH1A,ADH1B,ADH1C,ADH5,ALDH3A1,CYP \\
\hline metabolism & - & & 1A2,CYP2A6,CYP2B6,CYP2C19,CYP2C8,CYP2 \\
\hline cytochrome & & & C9,CYP2D6,CYP2E1,CYP3A4,CYP3A5,ENSG0 \\
\hline \multirow[t]{6}{*}{$\mathrm{P} 450$} & & & 0000270386,GSTA1,GSTM1,GSTO1,GSTO2,GS \\
\hline & & & TP1,GSTT2B,HPGDS,MGST1,MGST2,MGST3, \\
\hline & & & UGT1A1,UGT1A10,UGT1A3,UGT1A4,UGT1A5 \\
\hline & & & ,UGT1A6,UGT1A7,UGT1A8,UGT1A9,UGT2A2, \\
\hline & & & UGT2A3,UGT2B10,UGT2B11,UGT2B15,UGT2 \\
\hline & & & B17,UGT2B28,UGT2B4,UGT2B7 \\
\hline Retinol & 32 & 8.99E-39 & ADH1A,ADH1B,ADH1C,ADH5,CYP1A1,CYP1 \\
\hline \multirow[t]{6}{*}{ metabolism } & & & A2,CYP2A6,CYP2B6,CYP2C18,CYP2C8,CYP2 \\
\hline & & & C9,CYP3A4,CYP3A5,ENSG00000270386,UGT1 \\
\hline & & & A1,UGT1A10,UGT1A3,UGT1A4,UGT1A5,UGT \\
\hline & & & 1A6,UGT1A7,UGT1A8,UGT1A9,UGT2A2,UGT \\
\hline & & & 2A3,UGT2B10,UGT2B11,UGT2B15,UGT2B17, \\
\hline & & & UGT2B28,UGT2B4,UGT2B7 \\
\hline Steroid & 30 & $2.17 \mathrm{E}-36$ & AKR1C1,AKR1C2,AKR1C3,AKR1C4,CYP1A1, \\
\hline
\end{tabular}




\begin{tabular}{|c|c|c|c|}
\hline hormone & & & CYP1A2,CYP1B1,CYP2E1,CYP3A4,CYP3A5,E \\
\hline biosynthesis & & & NSG00000270386,HSD11B1,UGT1A1,UGT1A1 \\
\hline & & & 0,UGT1A3,UGT1A4,UGT1A5,UGT1A6,UGT1A \\
\hline & & & 7,UGT1A8,UGT1A9,UGT2A2,UGT2A3,UGT2B \\
\hline & & & 10,UGT2B11,UGT2B15,UGT2B17,UGT2B28,U \\
\hline & & & GT2B4,UGT2B7 \\
\hline Drug & 31 & $3.65 \mathrm{E}-35$ & CYP2A6,CYP2E1,CYP3A4,ENSG00000270386, \\
\hline metabolism - & & & GSTA1,GSTM1,GSTO1,GSTO2,GSTP1,GSTT2 \\
\hline other enzymes & & & B,MGST1,MGST2,MGST3,UGT1A1,UGT1A10, \\
\hline & & & UGT1A3,UGT1A4,UGT1A5,UGT1A6,UGT1A7, \\
\hline & & & UGT1A8,UGT1A9,UGT2A2,UGT2A3,UGT2B10 \\
\hline & & & ,UGT2B11,UGT2B15,UGT2B17,UGT2B28,UGT \\
\hline & & & 2B4,UGT2B7 \\
\hline Pentose and & 22 & $3.05 \mathrm{E}-28$ & AKR1A1,AKR1B1,ENSG00000270386,KL,UGT \\
\hline glucuronate & & & 1A1,UGT1A10,UGT1A3,UGT1A4,UGT1A5,UG \\
\hline interconversion & & & T1A6,UGT1A7,UGT1A8,UGT1A9,UGT2A2,UG \\
\hline $\mathrm{S}$ & & & T2A3,UGT2B10,UGT2B11,UGT2B15,UGT2B17 \\
\hline & & & ,UGT2B28,UGT2B4,UGT2B7 \\
\hline Ascorbate and & 20 & $1.50 \mathrm{E}-26$ & ALDH9A1,ENSG00000270386,UGT1A1,UGT1A \\
\hline aldarate & & & 10,UGT1A3,UGT1A4,UGT1A5,UGT1A6,UGT1 \\
\hline
\end{tabular}


metabolism

Metabolic

pathways

65
A7,UGT1A8,UGT1A9,UGT2A2,UGT2A3,UGT2

B10,UGT2B11,UGT2B15,UGT2B17,UGT2B28,

UGT2B4,UGT2B7

3.44E-26 ADH1A,ADH1B,ADH1C,ADH5,ADPGK,AKR1

A1,AKR1B1,AKR1C3,AKR1C4,ALDH3A1,ALD

H9A1,CBR1,CBR3,CYP1A1,CYP1A2,CYP24A1

,CYP27A1,CYP27B1,CYP2A6,CYP2B6,CYP2C1

8,CYP2C19,CYP2C8,CYP2C9,CYP2E1,CYP2R1

„CYP3A4,CYP3A5,DHFR,DHFRL1,ENSG00000

270386,GCH1,GCLC,GGT1,GSS,GSTZ1,HPGD

S,HSD11B1,KL,MCEE,PCK1,PRDX6,PTGES,PT

GES2,PTGES3,PTS,SPR,UGT1A1,UGT1A10,U

GT1A3,UGT1A4,UGT1A5,UGT1A6,UGT1A7,U

GT1A8,UGT1A9,UGT2A2,UGT2A3,UGT2B10,

UGT2B11,UGT2B15,UGT2B17,UGT2B28,UGT

2B4,UGT2B7

Glutathione $\quad 20 \quad 1.62 \mathrm{E}-22$ GCLC,GGT1,GPX1,GPX2,GPX3,GPX4,GPX7,G

metabolism
PX8,GSR,GSS,GSTA1,GSTM1,GSTO1,GSTO2,

GSTP1,GSTT2B,HPGDS,MGST1,MGST2,MGS

$\mathrm{T} 3$ 


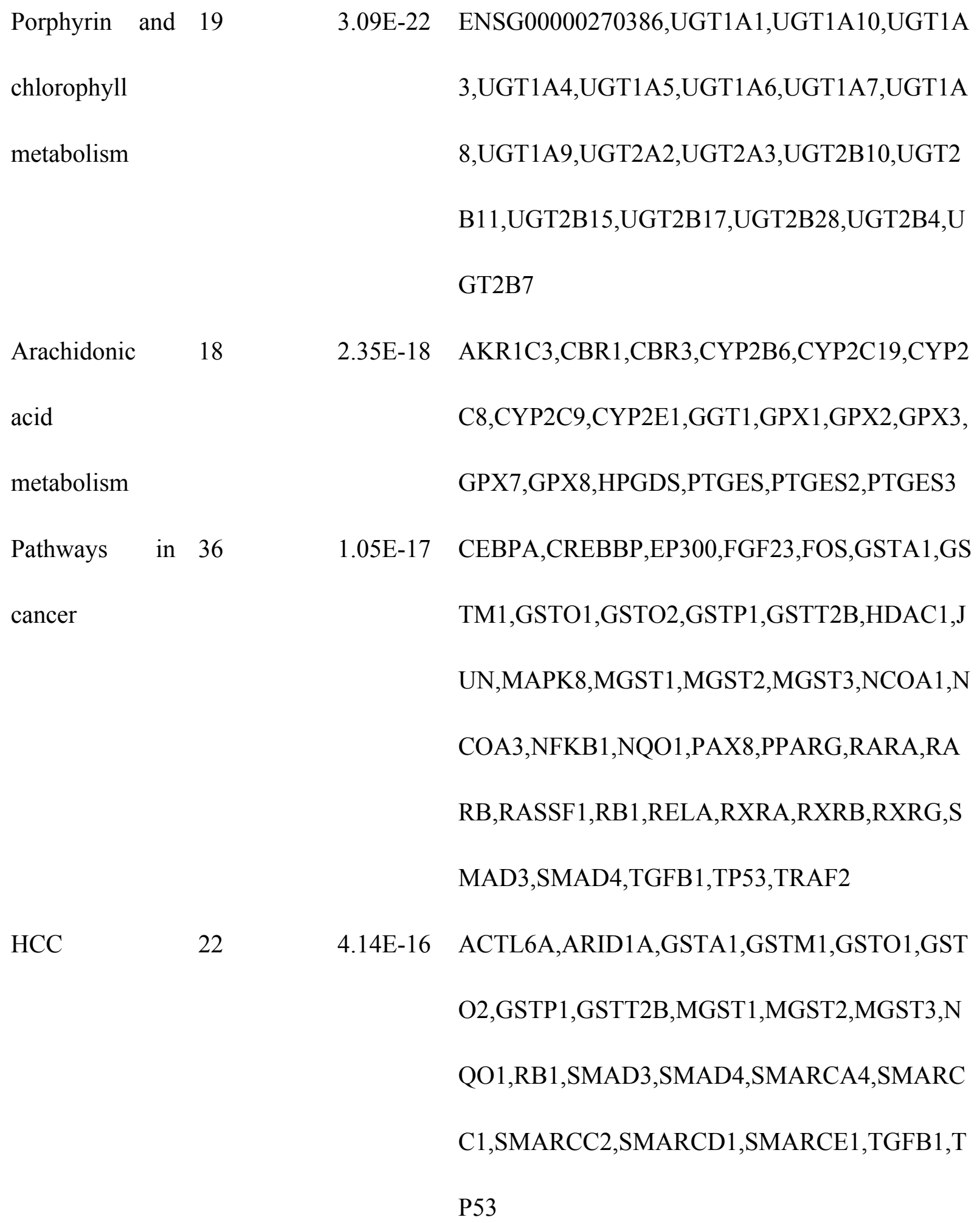

Fluid shear $19 \quad 2.45 \mathrm{E}-14$ FOS,GSTA1,GSTM1,GSTO1,GSTO2,GSTP1,GS 
stress

andatherosclero

sis

2 FDR, false discovery rate
TT2B,JUN,MAPK8,MGST1,MGST2,MGST3,NF

KB1,NQO1,PIAS4,RELA,SUMO2,TNF,TP53 


\section{Table 4 (on next page)}

\section{Table 4}

Mutual exclusivity analysis of 5 genes (TP53, RB1, TGFB1, GSTP1, and GSTM1) in 7 studies associated with HCC 
1 Table 4. Mutual exclusivity analysis of 5 genes (TP53, RB1, TGFB1, GSTP1, and GSTM1) in 7

2 studies associated with $\mathrm{HCC}$

\begin{tabular}{|c|c|c|c|c|}
\hline $\mathrm{A}$ & $\mathrm{B}$ & Log2 Odds Ratio & p-Value & Tendency \\
\hline TP53 & RB1 & 0.755 & 0.024 & Co-occurrence \\
\hline RB1 & TGFB1 & 2.131 & 0.108 & Co-occurrence \\
\hline TP53 & GSTP1 & 1.045 & 0.187 & Co-occurrence \\
\hline TP53 & TGFB1 & 0.564 & 0.421 & Co-occurrence \\
\hline RB1 & GSTP1 & $<-3$ & 0.437 & Mutual exclusivity \\
\hline TP53 & GSTM1 & 1.299 & 0.495 & Co-occurrence \\
\hline RB1 & GSTM1 & $<-3$ & 0.861 & Mutual exclusivity \\
\hline TGFB1 & GSTP1 & $<-3$ & 0.918 & Mutual exclusivity \\
\hline GSTP1 & GSTM1 & $<-3$ & 0.979 & Mutual exclusivity \\
\hline TGFB1 & GSTM1 & $<-3$ & 0.985 & Mutual exclusivity \\
\hline
\end{tabular}

3 